\title{
Retour sur deux projets de développement en aquaculture outre-mer : leçons pour l'avenir
}

\author{
Denis Lacroix \\ Agronome $^{1}$, IFREMER, Direction scientifique, 34203 Sète cedex, France
}

À la croisée de la critique du développement et des processus d'innovation technologique, cet article constitue un document rare : un retour d'expérience sans fard, par l'innovateur lui-même, sur l'histoire pluridécennale des projets de développement de l'Outre-mer français à partir de l'aquaculture, parsemée autant d'échecs que de réussites. Il illustre la difficulté de mettre en place un véritable processus d'apprentissage, et explore l'utilité mais aussi la résistance des concepts de la sociologie de l'innovation pour qui veut formuler des recommandations pour l'action.

La Rédaction

\section{Mots-clés :}

développement durable ;

gouvernance ; aquaculture ; innovation ; acceptabilité sociale

\section{Keywords:}

sustainable development; governance; aquaculture; innovation; social acceptability

Résumé - De nombreux projets d'aquaculture ont été menés depuis les années 1970 dans les territoires et départements français d'outre-mer. L'objectif était de diversifier l'agriculture avec des productions destinées au marché local comme aux Antilles, ou à l'exportation comme en Guyane. L'étude s'intéresse au bilan de deux projets d'élevage de la crevette tropicale d'eau douce aux Antilles et en Guyane, plus de vingt ans après leur lancement. L'objectif est de comprendre comment des innovations technologiques pertinentes ont conduit à deux échecs sur le moyen terme et de tirer des recommandations de méthode afin de réduire les risques d'erreurs ultérieures. Pour cela, deux cadres d'analyse sont mobilisés : le premier s'appuie sur les concepts de temporalité, légitimité et acceptabilité ; le second, motivé par la recherche subjective de cadres explicatifs, emprunte la vision de la théorie de l'acteur-réseau. Les trois déterminants majeurs du succès ou de l'échec se révèlent être la réactivité des responsables face aux problèmes d'adaptation à l'évolution des marchés, la qualité du transfert du leadership lors du passage de la phase de lancement à la phase de croisière et enfin la capacité de prendre en compte dès le départ les conséquences des scénarios d'évolution du projet, y compris la réussite technique. Une capacité d'intégration de ces types d'analyse, dans un cadre exante, devrait permettre de réduire les risques d'échec dans des projets de développement similaires à venir.

\begin{abstract}
Lessons for the future from two development projects in aquaculture in the French overseas territories. Numerous research and development projects have been launched since the seventies in the French overseas territories. Their aim was to foster new activities in agriculture based on local productions for local markets, notably in the French West Indies (FWI). In this paper we analyze two development projects on the rearing of giant freshwater prawn, one in the FWI and the other in French Guyana, over twenty years after their launching. The purpose of these compared studies is twofold: first to try to understand why a set of relevant technical innovations ended in failure 15 years later; second to identify recommendations in terms of methodology in order to diminish risks of mistakes or failure for future projects. To reach this goal we followed two approaches. A first analysis is based on a set of three criteria: social acceptability, legitimacy and time planning. The second analysis, justified by the subjective need for an explanatory tool, is based on the actor-network theory. The results show that beyond the levels of political support and financial investment and support the three key criteria for success are: (i) reactivity of the decision-makers, notably when markets change, (ii) quality of leadership transfer between the launching phase and the stabilized phase, and (iii) capacity of the decision-makers to take into account the concrete consequences of the various scenarios. Regarding this third criterion, our analysis suggests that the scenario of full technical success needs to be explored, notably in terms of economic sustainability. The integration of these two types of analyses into an ex-ante frame should contribute to reducing risks of failure in similar development projects.
\end{abstract}

\footnotetext{
Auteur correspondant : dlacroix@ifremer.fr

1 Denis Lacroix est animateur de la réflexion prospective à l'Ifremer et spécialisé en aquaculture, analyse de projets et relations internationales. Il enseigne dans plusieurs universités.
} 


\section{Introduction}

La création en France du Centre national pour l'exploitation des océans (Cnexo) en 1970 a ouvert un immense champ de recherches en sciences marines notamment outre-mer. L'aquaculture représentait alors une promesse de développement pour les départements et territoires d'outre-mer (Dom-Tom), sur le modèle de ce qui était observé dans de nombreux pays, surtout en Asie. C'est pourquoi le Cnexo a décidé de lancer des recherches sur l'élevage de plusieurs espèces de crevettes à partir du Centre océanologique du Pacifique, à Tahiti. Deux familles d'espèces y étaient étudiées : les crevettes marines Pénéides et la crevette géante d'eau douce Macrobrachium rosenbergii. L'objectif était de mettre au point des méthodes d'élevage fiables, en écloserie (phase larvaire) comme en bassin (grossissement) pour en effectuer le transfert dans d'autres Dom-Tom. En effet, dans toutes les îles tropicales françaises se présentait déjà la nécessité de diversifier les activités agricoles traditionnelles (canne, banane). L'élevage de cette espèce apparaissait donc comme une voie prometteuse de développement avec des espèces voisines déjà connues localement et faciles à valoriser en termes d'image comme de filière.

De 1973 à 1977, la méthode d'élevage larvaire de M. rosenbergii a été mise au point à Tahiti (Aquacop, 1977). Le contrôle de tous les paramètres d'élevage et une densité larvaire élevée donnaient deux avantages à cette méthode: une excellente productivité et une grande fiabilité. C'est pourquoi elle a été choisie pour lancer des programmes d'aquaculture aux Antilles (dès 1979) et en Guyane française (dès 1983). Ces deux programmes ont ainsi bénéficié d'une innovation technologique majeure pour une filière neuve dans un contexte de fort soutien financier et politique. Ils ont tous deux échoué au bout d'une quinzaine d'années d'efforts. Aussi, il semble utile de tenter leur analyse afin d'en tirer les leçons notamment à l'heure d'un nouveau plan de développement de cette filière en Guyane (Anon, 2008) et d'ambitieux projets de pisciculture marine à Mayotte. Il s'agit donc d'un retour réflexif sur une pratique du développement telle qu'elle a été mise en œuvre par les décideurs politiques à l'échelle nationale (ministère de la Recherche, secrétariat d'État aux Dom-Tom), comme à l'échelle locale (conseils régionaux).

Le cadre général est celui du développement au sens global du terme: "L'ensemble des processus sociaux induits par des opérations volontaristes de transformation d'un milieu social, entreprises par le biais d'institutions ou d'acteurs extérieurs cherchant à mobiliser ce milieu, et reposant sur une tentative de greffe de ressources, de techniques ou de savoir » (Olivier de Sardan, 1995). Il s'agit ici à la fois de la problématique de l'introduction d'une innovation (les méthodes d'élevage de la crevette d'eau douce) dans un milieu vierge de toute activité d'aquaculture et de celle de la socioanthropologie du contexte qui la reçoit.

En tant que responsable du lancement de cette filière aquacole aux Antilles françaises, de 1979 à 1984, puis comme adjoint de J.M. Griessinger, pour la même fonction, en Guyane française, de 1985 à 1991, soit l'année de la fermeture de l'écloserie régionale, j'ai toujours voulu tenter d'établir les raisons de ce double échec. Dans cette étude, il s'agit là moins de détailler la chronologie des actions et les responsabilités afférentes que de comprendre les mécanismes à l'œuvre pour réduire les risques d'échecs dans de futurs projets d'aquaculture, notamment outre-mer. Dans cette optique, il m'a paru intéressant de rechercher des approches contemporaines de ces projets afin de voir si l'on aurait pu, à l'époque, mieux éclairer les choix techniques et économiques. Le processus présenté ici est donc la construction, par l'innovateur qui était alors responsable du développement des projets, d'une sorte de boîte à outils à la fois opérationnelle et pragmatique, permettant d'esquisser le cadre d'un bilan ex post qui n'a jamais été fait ${ }^{2}$.

La première approche est centrée sur la dynamique de l'innovation elle-même, avec la succession des cinq types d'adoptants : pionniers, innovateurs, majorité précoce, majorité tardive, retardataires (Rogers, 1983; Mendras et Forsé, 1983). Cette vision de la problématique, qui date des années 1980, et est donc contemporaine des projets concernés, met l'accent sur l'importance de l'appropriation, du temps, de la valeur spécifique de l'innovation liée à ses propriétés (avantages, compatibilité, facilité d'appropriation, risques, etc.), des relations avec les acteurs qui s'y intéressent, au sein de structures sociales données. Elle pourrait être résumée en trois axes d'analyse : temporalité, au double sens d'opportunité et de planification, légitimité de l'innovation comme des acteurs, acceptabilité de l'innovation comme des jeux de pouvoir.

La seconde approche est celle des multirationalités, dans le cadre des systèmes de valeurs (vision du monde, normes, coutumes, etc.). Elle se déploie principalement dans l'analyse des réseaux d'acteurs et de leurs interactions avec des effets de clientèle et de pouvoir. Selon les régions étudiées, les combinatoires sont chaque fois nouvelles, avec des logiques politiques, économiques, voire symboliques différentes. Dans cette perspective, le cadre de la sociologie de la traduction (Callon, 1986) apparaît pertinent pour essayer de mettre en évidence les interactions entre les acteurs et comprendre la dynamique du système jusqu'à son extinction.

2 Ce travail reprend des éléments d'une thèse en sciences animales de D. Lacroix, réalisée au sein de l'école doctorale Abies (AgroParisTech) en 2010 sur « Aquaculture et prospective». 
Les grilles de relecture de ces deux histoires d'échecs $\mathrm{du}$ développement visent à identifier les dimensions à prendre en compte dans l'analyse d'une situation d'innovation pour répondre à des questions comme cellesci : pourquoi, en Guyane, n'a-t-on pas cherché à poser dès le début du programme la question-clé des marchés ? Est-ce qu'une simple analyse économique aurait permis de voir dès le début que l'objectif de l'exportation était une impasse?

Ces cadres d'analyse, construits pour cette analyse ex post à partir d'une inspiration par deux courants distincts dans la sociologie de l'innovation, doivent être d'abord précisés afin que leur portée comme leurs limites soient identifiées. Ils seront ensuite mobilisés dans l'étude des deux cas proposés, aux Antilles françaises, puis en Guyane. Ensuite, leur pertinence sera évaluée en termes d'interprétation de ces deux « histoires » d'innovation et en termes de valeur pédagogique pour l'avenir. En effet, l'intérêt de s'interroger sur les paramètres des projets et surtout sur le processus de leur gestion est d'éviter la répétition des erreurs dans d'autres projets de développement de l'aquaculture à forte incitation politique, comme c'est le cas général dans l'Outre-mer français.

\section{Cadres d'analyse}

\section{Temporalité, légitimité, acceptabilité}

Le premier cadre d'analyse (dit « TLA » pour temporalité, légitimité, acceptabilité) mobilise trois critères qui apparaissent comme "cardinaux » dans le développement d'une innovation en aquaculture : la temporalité, la légitimité et l'acceptabilité, composants que l'on retrouve à différentes pondérations dans la bibliographie sur les aspects de méthodologie du développement (Pollnac et al., 1982 ; UICN, 2007 ; GFCM, 2010) comme dans l'approche par l'analyse prospective (Schwartz, 1991 ; Godet, 1997). Cette approche permet de tenir compte de l'environnement naturel des projets comme du contexte socioéconomique (Passet, 1979 ; Mermet, 2005).

Le critère de la temporalité s'inscrit dans une double question : dans quel processus de long terme et à quel stade s'inscrit l'innovation? Un élu ou un entrepreneur imaginent une situation qui pourrait advenir dans une région et un contexte donnés, dans un secteur jugé riche de potentiel, selon des conditions jugées a priori réalisables. H. de Jouvenel (2004) souligne l'importance de la prise en compte du temps long dans toute action de développement. Celle-ci doit intégrer les trois composantes classiques de l'analyse d'une problématique sur le long terme : tendances lourdes, germes de changement et points de rupture. Si la première composante est souvent bien documentée, parce que les tendances s'enracinent dans des données et des analyses du passé, les deux autres composantes sont traitées souvent de manière plus vague ou incomplète par les experts alors qu'elles sont critiques pour le projet (Alcamo, 2008).

Second aspect à prendre en compte : la dynamique du développement mis en place reste-t-elle pertinente si des ruptures sont identifiées ? Ainsi, en aquaculture, si la contrainte majeure des îles tropicales devient celle des surcoûts liés à leur isolement, est-il toujours justifié de soutenir des élevages à vocation d'exportation?

Enfin, la temporalité s'inscrit dans la logique de la conduite de projets puisque le bon «tempo » des financements, comme de la mobilisation des compétences, fait partie de la gouvernance au même titre que la légitimité des acteurs ou l'acceptabilité du projet auprès des parties prenantes.

La légitimité du porteur de l'innovation est au cœur de la gouvernance d'un projet parce qu'elle conditionne la position des acteurs au moment de la phase initiale, la plus sensible. Il s'agit là « d'un processus de coordination d'acteurs, de groupes sociaux et d'institutions pour atteindre des buts discutés collectivement » (Le Galès, 2006). De fait, la plupart des projets européens de recherche et développement en aquaculture s'inscrivent dans une démarche de gestion intégrée. Dans celle-ci, le groupe porteur du projet doit être « reconnu par tous et avoir les capacités nécessaires de coordination et d'intégration » (Hénocque et Billé, 2005).

Dans le cas de l'aquaculture en France, c'est l'État qui détient le plus souvent la légitimité de l'initiative. La difficulté récurrente réside alors dans le passage de relais entre l'État et un ou plusieurs porteurs du projet parce que ce transfert de légitimité révèle souvent des motivations cachées ou des objectifs de long terme différents des buts affichés au départ du projet (Cornish, 2004).

Les choix des parties prenantes, comme des dispositifs institutionnels de concertation ou de financement, posent aussi des problèmes de légitimité (Claes-Mekdade, 2006) et d'évaluation des risques (Chevassus-au-Louis, 2007). Ces questions justifient un investissement important dans l'explicitation initiale des buts poursuivis, des méthodes employées et du rôle des partenaires (Jamieson, 2005). Le critère de l'acceptabilité est par nature large. Dans quelle mesure le tissu social devant accueillir l'innovation est-il prêt à la recevoir ? Cette question porte sur le tissu des entrepreneurs auxquels l'innovation peut être transférée, les consommateurs potentiels, et la société locale, «cible » du développement. Cette dynamique pose des problèmes récurrents d'appropriation et d'apprentissage (Van der Heijden, 1996). Ce concept d'acceptabilité, mot d'ingénieur pour 
faire accepter «son projet» au profane ${ }^{3}$, est parfois contesté comme appartenant à une vision «top-down" des processus d'innovation. Il est mentionné ici comme un des deux éléments du couple "Légitimité du porteur de projet/Acceptabilité par le tissu social ». L'analyse de ce binôme facilite ensuite la description du jeu des acteurs engagés dans le processus d'innovation (Alter, 2000).

Des études réalisées dans des domaines divers (crise de la vache folle, déchets nucléaires) montrent que les «nœuds» des problèmes sont bien plus liés aux contextes et aux institutions chargées de la question qu'au problème lui-même (Marris, 2001). Le mauvais positionnement initial des débats est très coûteux en termes d'image comme de temps et d'énergie nécessaires pour corriger celle-ci quand elle est fausse, même quand elle est portée par une autorité légitime (Schwartz, 1991).

Dans le cas de tout projet innovant, une des clés de l'acceptabilité est dans le partage précoce de l'information et des raisons des choix techniques (Dherse et Minguet, 1986). Les controverses impliquant tous les acteurs permettent d'explorer les débordements engendrés par le développement des sciences et des techniques. «Elles réalisent un inventaire de la situation [...] qui la rend intelligible. Elles constituent donc un enrichissement de la démocratie » (Callon et al., 2001). Autrement dit, «ce que demandent nos concitoyens, ce n'est pas le risque zéro, mais le mépris zéro " (Noiville et Gouyon, 2000).

\section{Appui du cadre de la théorie de l'acteur-réseau}

Le premier cadre d'analyse évoqué (TLA) présente l'inconvénient de mal prendre en compte la dynamique générée par une innovation. Il est donc intéressant d'étudier le système et son évolution en s'inspirant des concepts proposés par Callon (1986) et structurés dans le cadre de la théorie de l'acteur-réseau. Cette théorie s'enracine dans un "cas fondateur » issu de l'aquaculture. Sociologue, M. Callon s'est intéressé à l'aquaculture dès les années 1980, au moment où il s'agissait d'un domaine largement expérimental et donc controversé. Il a étudié les débats entre chercheurs et pêcheurs en baie de Saint-Brieuc sur les possibilités d'aménagement de la pêcherie locale de coquille Saint-jacques. Cette théorie, aussi connue par l'acronyme ANT (Actor Network Theory), permet d'élargir le regard par rapport au cadre précédent selon trois principales perspectives. D'abord,

\footnotetext{
3 On notera que l'étymologie oppose ici l'expert (qui est instruit par l'expérience) au profane (qui se tient pro-fanum, « hors du temple »), d'où une certaine « sacralisation » de celui qui sait, et se trouve donc dans le « temple » du savoir par opposition à l'ignorant qui se trouve en dessous du temple (Dictionnaire Le Petit Robert, 1984).
}

elle prend en compte comme " actants » non seulement les humains mais aussi les objets et les discours (politiques, techniques, etc.) via leurs porte-parole. Or, dans les deux cas étudiés, le discours politique du transfert de la recherche au développement joue un rôle central. Ensuite, elle étudie l'ensemble des relations qui les font évoluer au sein d'un réseau de traductions croisées. Là encore, aux Antilles comme en Guyane, le nombre des actants est élevé et les discours sont le plus souvent biaisés par la volonté de prouver, y compris celui des scientifiques. Enfin, elle montre comment la problématisation révèle le ou les points de passage obligés des actants : une carte d'implantation des fermes à l'échelle d'un territoire, une écloserie coopérative... Il apparaît particulièrement pertinent pour relire les cas d'étude de noter que, dans cette théorie, trois phénomènes font évoluer le système : les dispositifs d'intéressement ou d'alliance entre actants, la mobilisation des alliés par les porte-parole avec des niveaux de transparence variables, et enfin les controverses qui remettent le système en fluctuation jusqu'à un nouvel équilibre, ou son éclatement, ou la naissance d'une innovation.

Il faut rappeler que l'aquaculture s'est développée rapidement depuis soixante ans : elle est passée d'environ 5 millions de tonnes en 1950 à 52 millions de tonnes en 2008 (FAO, 2010). D'une situation modeste dans l'alimentation humaine, le poisson est devenu la quatrième source de protéines élevées dans le monde, après le bœuf, le poulet et le porc (FAO, 2008). Cette jeunesse du secteur explique que tout projet d'aquaculture engendre interrogations et polémiques autant qu'enthousiasme et créativité. Cette situation justifie l'appel à la sociologie de la traduction autant pour l'analyse du passé que pour la projection dans le futur. En effet, c'est bien le processus de traduction qui est au cœur des problématiques et de leur transformation: traduction de connaissances scientifiques en projets de développement, traduction des attentes des acteurs via les porte-parole, traduction des discours en programme d'actions, "trahison» de certains acteurs déclenchant controverses, changements ou ruptures.

Mais en quoi cet outil permettrait-il d'aller au-delà $d^{\prime}$ 'une analyse classique de type SWOT ${ }^{4}$ ou d'un benchmarking, voire d'une étude de marché fondée sur des indicateurs économiques classiques (Barré, 2000) ? Si l'on se réfère aux rares documents encore disponibles dans la littérature grise et traitant des questions de développement de l'aquaculture aux Antilles et en Guyane, on observe que la demande est d'abord d'origine politique : le conseil régional aux Antilles et l'État en

4 SWOT pour Strengths, Weaknesses, Opportunities and Threats, tableau des "forces, faiblesses opportunités et menaces » concernant tout projet dans les années 1980 (Boston Consulting Group). 
Guyane. Les données économiques censées justifier les investissements mettent en avant le prix élevé du Macrobrachium sur les marchés et donc la forte rentabilité potentielle de son élevage (Lacroix et al., 1987 ; Lacroix et Maubras, 1991). Mais ce qui préoccupe les décideurs est moins l'élasticité des prix que la fiabilité de l'écloserie parce que celle-ci n'a pas encore fait la preuve de son efficacité à une échelle industrielle. Il s'agit donc bien d'une problématique initiale de l'innovation (une écloserie moderne de crevettes) dans un milieu rural plutôt traditionnel. L'absence de mobilisation des analyses économiques et stratégiques standard ne peut donc pas se comprendre sans mettre en évidence les processus sociotechniques que les deux cadres envisagés ici cherchent à éclairer dans une visée de conseil opérationnel. Ces deux approches analytiques ont ainsi pour objet d'aider à une vision "stéréoscopique » des deux projets étudiés afin de comprendre la mécanique de leur échec et d'en extraire des recommandations de méthode pour réduire les risques d'erreurs ultérieurs dans des projets similaires.

\section{Antilles}

\section{Approche « temporalité, légitimité, acceptabilité »}

Dans les Antilles françaises, les projets abondent pour tenter de diversifier l'agriculture, trop dépendante de monocultures (canne à sucre puis banane) dans un contexte d'urbanisation rapide. La filière aquacole part de zéro en 1977. Sa mise en place suit quatre phases: identification d'un marché solvable, maîtrise de l'écloserie et de l'élevage en bassin, développement des fermes en nombre et en taille, organisation de la filière à l'arrêt des subventions. Impressionné par la réussite de cette filière lors de sa visite à l'île Maurice en 1977, le viceprésident du conseil régional de Martinique, Jean Bally, fait appel en 1979 au Centre national pour l'exploitation des océans (Cnexo) pour transférer la technologie mise au point à Tahiti.

La légitimité est portée ici par les élus locaux qui cherchent de nouveaux produits agricoles, peu délocalisables. L'élevage de crevettes paraît ainsi d'autant plus justifié qu'il offre un produit proche de la crevette d'eau douce locale ${ }^{5}$, connue et appréciée, et qu'il peut être pratiqué en complément d'autres activités agricoles.

L'acceptabilité de ce projet par les acteurs est variable. Si l'État et les collectivités locales soutiennent la filière, certaines organisations professionnelles restent méfiantes pour diverses raisons: concurrence dans l'attribution d'aides, captages supplémentaires d'eau,

\footnotetext{
5 Dite «Ouassous », prononciation en créole local de « Roi des sources ».
}

pollution organique, etc. Le grand public est intéressé parce que cette nouvelle espèce que l'on tente d'élever ressemble à l'espèce locale, surpêchée, donc chère.

\section{Approche par la théorie de l'acteur-réseau}

Il faut noter d'abord que les actants ${ }^{6}$ à prendre en compte sont nombreux: élus, éleveurs, scientifique (innovation), techniciens, technologie (écloserie), crevettes, marchés, bassins d'élevage et grand public. Dans cette liste qui permet de mettre au même niveau tous les « moteurs » d'action, le «bien commun », clé du développement, est l'écloserie ; en effet, sans cet outil, pas de production de masse fiable de post-larves (PL), condition sine qua non de l'investissement des éleveurs dans des bassins. La figure 1 illustre la complexité des interactions des actants dans le réseau et leurs liens avec l'enjeu central. Le marché est unique et local car il y a 400000 habitants dans l'île sans compter le tourisme.

La question des porte-parole et de leur légitimité paraît initialement simple parce que les acteurs sont bien visibles : le chef de file des « politiques » est J. Bally, porteur de l'idée initiale ; les éleveurs sont tous dans la coopérative $\left(\mathrm{Sica}^{7}\right)$ animée par un président consensuel ; les techniciens sont gérés par une société d'études locale ; le marché initial est local avec vente à la ferme et par la Sica jusqu'à l'arrivée des « grandes » fermes ; les médias sont réduits au journal local (France-Antilles); et le scientifique est seul.

La dynamique du système va évoluer en six ans par déblocage de verrous successifs : la démonstration de la fiabilité et de l'efficacité de la méthode « eau claire », le doublement en quatre ans des rendements en grossissement, la formation à tous niveaux, la réponse positive du marché local, l'agrandissement de l'écloserie, l'apparition de "grandes » fermes de plusieurs hectares.

Autour de l'écloserie se construit l'unité (relative) des éleveurs. La propriété de l'écloserie est transférée en 1982 du conseil régional à la Sica qui gère aussi l'assistance technique aux éleveurs et la commercialisation de la majeure partie de la production. Le jeu des alliances est simple : chaque groupe d'acteurs cherche à valoriser son rôle auprès du conseil régional pour renforcer sa position et pérenniser aides et emplois. La position du chercheur est celle de la " science impartiale » au service de tous, sur demande des politiques. J'ai donc été « enrôlé » par les élus, en qualité informelle de « conseiller scientifique », ce qui m’a conféré ma légitimité, a renforcé mon image de position d'impartialité et

\footnotetext{
6 Dans la suite du texte, j'emploie le terme d'actant pour tous les éléments du réseau, humains ou non. Quand il ne s'agit que de personnes ou de groupes humains, j'emploie alors le terme d'acteur.

7 Société d'intérêt collectif agricole.
} 


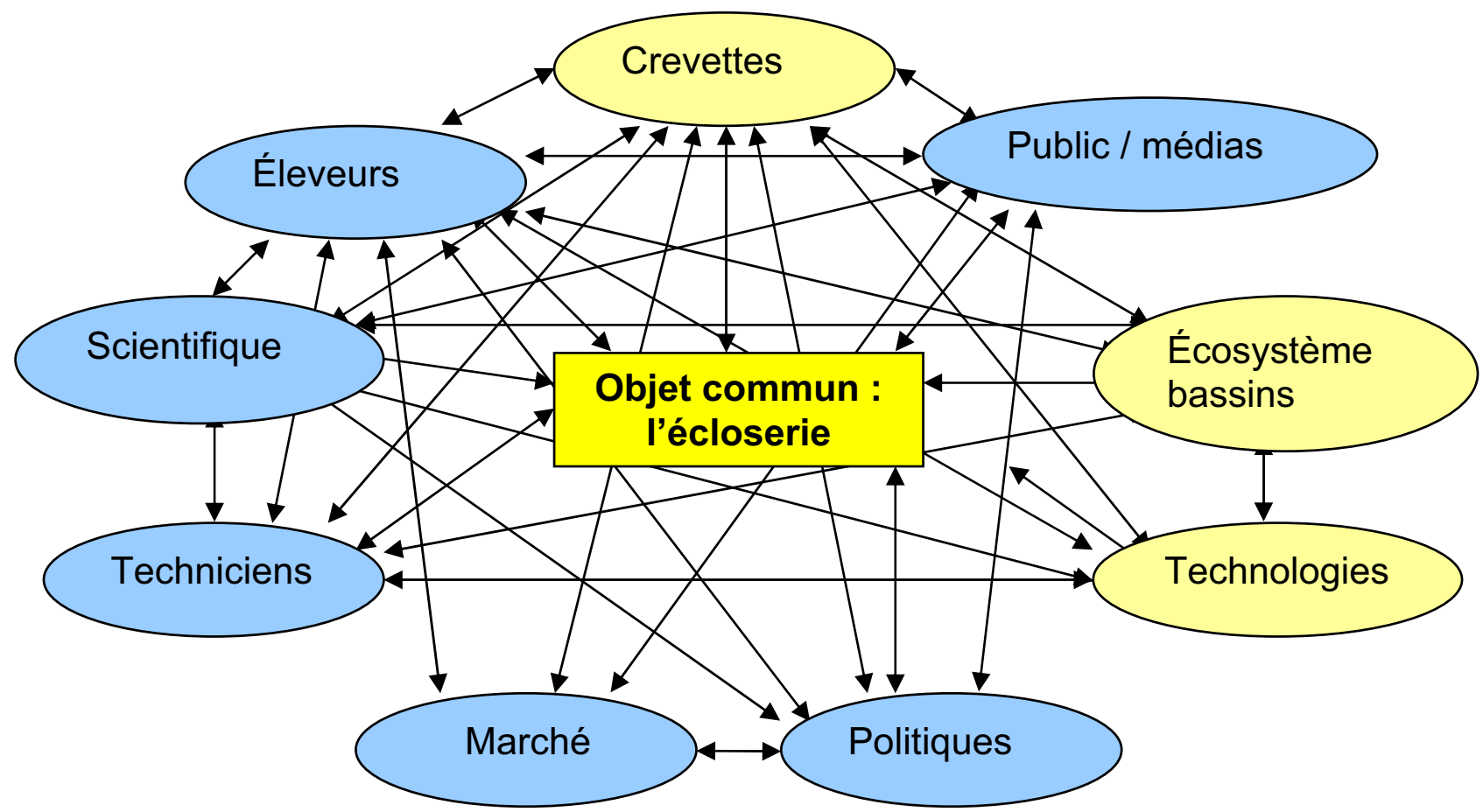

Fig. 1. Schéma du réseau d'actants appliqué au cas du développement de l'aquaculture de la crevette d'eau douce Macrobrachium rosenbergii aux Antilles françaises de 1976 à 1990.

m'a mis à l'abri de deux types de pressions : celles de certains éleveurs, qui m'auraient volontiers recruté comme conseiller technique permanent, et celles de la société d'aide technique locale, chargée de la gestion du projet, et qui aurait préféré que je sois un cadre mis à sa disposition.

Les composantes techniques n'ont jamais constitué de frein au développement. L'écloserie « eau claire » a bien fonctionné dès son premier cycle et la nouvelle méthode de grossissement en système continu, mise au point sur place en deux ans, a été généralisée rapidement. L'assistance technique aux éleveurs et la progression régulière des rendements ont créé au départ une dynamique générale de confiance. Ce modèle «vertueux » est vite reproduit dans l'île de la Guadeloupe, dès 1982.

Mais deux changements vont modifier le système : le transfert de la responsabilité du développement à la Sica et la création de plusieurs écloseries artisanales, ce qui va entraîner un effet de dumping par rapport au coût de la post-larve (PL), qui est ensemencée dans les bassins de grossissement des éleveurs de l'écloserie régionale. Ce nouveau système, qui ne garantit aucune fiabilité d'apport en PL pour les éleveurs, contribue à affaiblir financièrement les Sica. Celles-ci ne traitent plus qu'un volume réduit de crevettes à vendre car un nombre croissant d'éleveurs préfèrent la vente directe, plus rentable. Enfin l'assistance technique et la formation ne peuvent plus être prises en charge parce qu'elles auraient dû être financées par des taxes sur la vente de $\mathrm{PL}$, en régression, et sur la vente mutualisée des crevettes, abandonnée.

Les filières sont donc mal armées pour affronter les deux chocs successifs de la concurrence de produits équivalents importés d'Asie (années 1980), et la baisse lente des rendements ${ }^{8}$ (années 1990). Elles ne disposent plus ni de subvention, ni d'une unité de représentation auprès des élus ou des pouvoirs publics, ni de vision de moyen terme. Elles sont donc sans capacité de réaction ou d'adaptation à des problèmes nouveaux. Chaque éleveur doit trouver des solutions personnelles. Au sens de la théorie ANT, après le «choc » du marché, le réseau des actants ne s'est pas recomposé. Le lien collectif central est donc perdu sans qu'il soit remplacé par un enjeu de construction collective comme aurait pu l'être la création d'un label de production locale par exemple, pour se distinguer de la production asiatique en congelé. Les petits éleveurs perdent toute rentabilité et beaucoup abandonnent l'activité. Il est intéressant de noter que cette évolution est exactement parallèle à celle observée pour le développement de la pisciculture à la même

\footnotetext{
8 Diverses études faites sur ce problème identifient plusieurs causes plausibles: consanguinité, envasement des bassins, nocivité des produits de traitement des bananes pour les arthropodes, dérive des méthodes de grossissement, etc.
} 
époque dans l'État brésilien de Sao Paulo, avec les mêmes atouts de départ, la même spirale vertueuse des premières années, puis le désengagement de l'État sans transition progressive, la déstructuration du réseau et l'abandon de la filière sauf pour quelques producteurs liés à des marchés de niche (Da Silva et al., 2005).

\section{Conclusion pour les Antilles}

Aux Antilles, le projet d'une écloserie « eau claire » est une innovation complète avec une prise de risque élevée. Le contexte est celui d'un système multiacteurs avec un leadership politique fort. L'objectif est clair et partagé, les budgets de mise en œuvre des moyens sont suffisants, le marché, attractif et la perception du public, positive. Les clés du développement sont, au départ, clairement d'ordre technique (écloserie et grossissement). Les deux phases du système sont vite jugées fiables par les éleveurs d'où la confiance générale dans le développement de cette filière.

Le dysfonctionnement majeur vient du mauvais transfert de responsabilités entre les « politiques » et les «producteurs » au moment où pourtant les écloseries fonctionnent bien et l'élevage en bassin atteint de bons rendements. Disposant de peu d'autorité comme de moyens, les Sica vont incarner de moins en moins la réalité du développement. Vu sous l'angle des processus décrits par la théorie ANT, tout semble se passer comme si le changement du porteur majeur du projet était perçu par certains acteurs comme la remise en cause du contrat moral qui les liait au système des actants. En effet, la «trahison» devient donc possible, voire légitime, puisque le cadre a changé. L'approche ANT révèle bien l'origine du dysfonctionnement : si les prix se stabilisent en raison de l'apparition sur le marché antillais de crevettes d'importation (du Vietnam, surtout), le consentement à payer plus cher pour un produit frais local valorisé par l'étiquette « Pays nous » reste stable. La viabilité économique des fermes n'a donc pas été menacée par une "guerre des prix » à la baisse pour contrer des importations. Finalement, la crevetticulture aux Antilles a survécu mais à l'échelle d'un marché de niche et sans durabilité.

\section{Guyane}

\section{Approche « temporalité, légitimité, acceptabilité 》}

En Guyane française, la légitimité du développement appartient à l'État pour des raisons historiques. Cette situation a été renforcée par deux phénomènes: le développement de la base spatiale de Kourou et l'accroissement des aides européennes aux régions ultrapériphériques (RUP) dont la Guyane. La dynamique liée au secteur spatial en Guyane ${ }^{9}$ justifie le soutien à l'agriculture locale, premier secteur d'emploi. La crevetticulture d'eau douce, composante du « Plan vert » de développement agricole, requiert une capacité de recherche finalisée car il s'agit d'une espèce importée ${ }^{10}$ dans un environnement atypique. En effet, l'écosystème de la forêt primaire est très différent de l'écosystème naturel du Macrobrachium. Il faut donc tester et adapter les méthodes de grossissement afin de fiabiliser les rendements au niveau de 2,5 t/ ha/an. Le programme, ambitieux, vise 200 ha de bassin soit $500 \mathrm{t}$ de production à terme.

L'arrivée en 1977 d'environ 500 réfugiés politiques Hmongs ${ }^{11}$ en Guyane apparaît comme un atout car ils s'insèrent rapidement dans le pays. Comme l'espèce Macrobrachium vient aussi de leur région d'origine, ils connaissent déjà cette crevette qui fait partie de leur cuisine traditionnelle. Aussi, l'État construit une ferme de 10 ha près de leur village, à Cacao, au bord du fleuve de La Comté, $70 \mathrm{~km}$ au sud-ouest de Cayenne.

Les défis à relever pour la filière Macrobrachium sont plus nombreux qu'aux Antilles car il faut sécuriser la production de masse de post-larves à une échelle bien supérieure à celle des Antilles, maîtriser l'écosystème «bassin de grossissement » et en préciser les méthodes de gestion afin d'assurer un rendement d'au moins $2 \mathrm{t} / \mathrm{ha} / \mathrm{an}$. En effet, les eaux douces issues du plateau des Guyanes sont très pauvres en minéraux et très acides ( $\mathrm{pH}$ de 3,5 à 5), en raison des processus de décomposition de la matière organique en forêt amazonienne. Dans un second temps, il s'agit d'organiser la commercialisation. Dans l'esprit des décideurs, l'ampleur de la tâche de la première phase est telle qu'il sera toujours temps de se préparer à la seconde. Une phrase résume bien la position des principaux décideurs, dans l'administration comme au sein des collectivités locales : "Essayez de produire d'abord; si vous y parvenez, on verra alors comment vendre... » Pour ma part, j'avais été surpris par cette position lors de mon arrivée en Guyane en 1985, mais le directeur départemental de l'agriculture m'avait assuré que la plupart des échecs de développement dans le domaine agricole étaient dus aux difficultés d'adaptation des technologies importées aux contraintes guyanaises, ce qui faisait que la question de la taille du marché n'avait pas le temps de se poser.

\footnotetext{
9 Comme le nucléaire militaire en Polynésie dans les années 1960 avait «justifié » la création du Centre océanologique du Pacifique.

10 À partir de post-larves exportées de l'écloserie de Martinique dont j'avais la responsabilité en 1980.

11 Communauté de réfugiés politiques des hauts plateaux vietnamiens et laotiens arrivés à la fin des années 1970 en Guyane après avoir transité plusieurs années par les camps de Thailande.
} 
Il faut reconnaître que le contexte est contraignant: un pays couvert à $90 \%$ de forêt primaire, des infrastructures limitées à la bande littorale, une population de 150000 habitants avec une forte pression d'immigration clandestine, des échanges impossibles avec les pays voisins, le Brésil et le Surinam ${ }^{12}$. La planification de tout projet de développement requiert ici plus de temps que dans les autres Dom-Tom. Sa temporalité est donc « étirée » en raison de multiples facteurs liés à l'éloignement, à la pénurie de personnel qualifié, à la médiocre qualité des infrastructures, aux aléas du climat (5 à $6 \mathrm{~m}$ de pluie/an), de la salinité de la mer (2 à $12 \%$ ) ou du faciès du littoral (bancs de boue récurrents). La volonté politique locale, qui rejoint celle de l'État, est de financer de nombreux projets sans beaucoup de conditions sur la faisabilité de long terme.

Un double dispositif d'aides au développement de l'agriculture (État et Union européenne) est géré localement, avec l'État comme opérateur central. Les collectivités locales et la chambre d'agriculture préparent, en concertation avec la direction départementale de l'agriculture (DDAF) et la préfecture, des programmes de développement à hauteur des financements escomptés. Ce dispositif crée des risques d'«effets d'aubaine» et attire des « chasseurs de prime».

Dans ces conditions, l'acceptabilité des projets de développement est élevée, ou du moins apparaît comme telle. Mais il faut distinguer celle-ci de l'implication réelle des acteurs. En effet, les premiers concernés sont les représentants de l'État qui ne restent pas au-delà de quatre années en Guyane en moyenne. Ils n'ont pas de point de comparaison antérieur en raison du caractère unique de celle-ci et, le plus souvent, faute d'un temps d'apprentissage des spécificités du pays, ils gèrent les affaires courantes sans investissement sur le long terme. Les autres acteurs souhaitent logiquement que le projet soit financé : agriculteurs, petites entreprises, coopératives agricoles, fournisseurs de matériel et d'intrants divers, structures mixtes comme le centre régional d'innovation technologique.

Cette situation est potentiellement risquée car il n'y a pas de système de critique autre qu'une évaluation ex post, si elle a lieu, et si elle s'intéresse non seulement aux stratégies de développement, mais aussi à la bonne exécution des budgets. Finalement, malgré une légitimité et une acceptabilité fortes du projet, conférées par la participation de tous les acteurs au processus de sélection des projets de développement, tout le monde a intérêt aux faux-semblants, comme c'est parfois le cas dans ce type de procédure (Blanc-Pamard et Fauroux, 2004). Par ailleurs, la «participation» des acteurs au

\footnotetext{
${ }^{12}$ À l'époque, le Surinam était en guerre civile et la frontière avec la France était fermée. Quant au Brésil, la route s'arrêtait à $100 \mathrm{~km}$ de la frontière marquée par le fleuve Oyapock.
}

projet de développement, valorisée depuis une quinzaine d'années (Lavigne Delville, 2011), notamment dans le domaine marin et celui de l'aménagement côtier (Rey-Valette et Roussel, 2006 ; UICN, 2007), peut être un leurre si elle ne repose pas sur une réelle volonté d'engagement des acteurs dans le projet (Kumar et Corbridge, 2002).

\section{Approche par la théorie de l'acteur-réseau}

Le cadre d'analyse identifié pour les Antilles est aussi complexe en Guyane. Apparaissent là deux objets collectifs (l'écloserie et les méthodes de grossissement) et deux actants majeurs : les marchés (local et international) et les infrastructures. Le moteur premier, les élus aux Antilles, est remplacé par l'État (préfecture de région et DDAF); enfin, scientifiques et techniciens peuvent être considérés dans le même groupe.

À la différence des Antilles, les éleveurs doivent être clivés en trois groupes :

1. La dizaine de petits éleveurs ( 3 à 5 ha chacun), dispersés sur $250 \mathrm{~km}$ le long de la route du littoral, de Cayenne à Saint-Laurent-du-Maroni.

2. La coopérative des Hmongs de Cacao (10 ha), en pleine forêt.

3. La Société aquacole de l'Orapu, ou Saor (37 ha), en quête permanente de son autonomie pour la commercialisation comme pour l'écloserie.

Si la phase d'écloserie a été bien maîtrisée, l'objet collectif majeur est la gestion du grossissement dans un environnement plutôt défavorable à l'espèce. Ainsi, la fiabilité d'une production de post-larves ne suffit plus à lancer seule le développement. Il a fallu adapter les méthodes de grossissement pour assurer le rendement moyen de $2 \mathrm{t} / \mathrm{ha} / \mathrm{an}$. Le nouvel actant, le marché international, n'a pas été identifié au départ, malgré l'ambition affichée du projet. La découverte de son rôle central, après la saturation du marché local, va bouleverser la position de tous les actants.

Dans ce cas encore, il est fait la démonstration de l'utilité des deux outils communs. Ainsi, grâce à une écloserie fiable, à vocation d'usage collectif, toutes les fermes, dont la Saor, peuvent ensemencer leurs bassins au temps prévu et avec les quantités recommandées. Cette première phase, conforme au plan, rassure tous les acteurs et renforce la crédibilité de l'ensemble du projet. Ensuite, les fermes, dont les rendements étaient très dispersés au départ faute de connaissances techniques, améliorent leurs résultats grâce aux méthodes préconisées par l'Ifremer. La maîtrise progressive du milieu d'élevage par les éleveurs et la diffusion in situ des techniques grâce à une assistance active ( 4 à 6 personnes) permettent d'accroître et de fiabiliser les rendements. J'ai pu mesurer personnellement l'évolution positive de la perception des actions de l'État (préfecture, DDAF et 
Ifremer) par les éleveurs lors des nombreuses visites d'assistance et de formation sur le terrain. Les Hmongs, récents citoyens français; les Japonais, spécialistes de l'élevage de crevettes ; les Hollandais, rêvant de « poldériser » les marais en face du port de Dégrad des Cannes ; les Guyanais, "chasseurs de primes »; les métropolitains, aussi intéressés par l'or du fleuve voisin que par la crevette, tous prennent confiance dans les deux objets communs dont les scientifiques démontrent la prévisibilité des progrès. Cependant, l'équilibre des porte-parole est moins stable qu'aux Antilles parce que l'État se borne à un rôle d'instructeur de dossiers et que les éleveurs restent divisés sur la stratégie de moyen terme. Les problèmes d'adaptation de la technologie étant réglés, en écloserie ${ }^{13}$ comme en grossissement, le jeu des « enrôlements » des acteurs va donner à terme quatre groupes :

1. L'État (via la DDAF) et les scientifiques vont devenir solidaires: il s'agit de tenir un discours commun de rigueur dans le respect des engagements de réalisation des travaux, de mise en eau des bassins, de paiement à l'écloserie des post-larves livrées, d'application des protocoles d'élevage et de participation à la gestion de la commercialisation.

2. La Sica des petits éleveurs défend sa survie mais avec des fermes dispersées.

3. La coopérative des Hmongs se méfie de cette structure collective qui lui rappelle ses débuts difficiles dans l'accès au marché local dans les années 1980 (Géraud, 1997).

4. La Saor est dirigée par Pierre Magnan, actionnaire majoritaire des Pêcheries de Guyane (Pideg); il pense que les Macrobrachium sont l'équivalent dulçaquicole des crevettes marines pêchées par ses chalutiers.

Les controverses vont porter sur la manière de traiter les problèmes de commercialisation parce que les producteurs sont vite en concurrence sur le petit marché local. Forte de son expérience en matière d'exportation de crevettes de pêche, la Saor décide de faire cavalier seul avec l'essai d'exportation du « Bouquet de Guyane » en France, sous forme de produit congelé. C'est un échec. Les partenaires, de moins en moins confiants les uns vis-à-vis des autres, ne croient plus en une solution collective.

La figure 2 fait apparaître la polarisation des liens, et donc le déséquilibre du système vers le binôme éleveurs - groupe scientifique. Dans un schéma de développement économique dépendant autant de subventions extérieures, on peut penser que le «copilote» du système avec les producteurs aurait dû être l'État, ou une structure consulaire représentative, capable de prendre

\footnotetext{
13 Il faut rappeler ici que malgré de nombreux aléas biologiques et techniques (chute de salinité de l'eau de mer, envasement à la prise d'eau, etc.), les livraisons des post-larves aux éleveurs n'ont jamais été retardées ou réduites.
}

en charge les problèmes de commercialisation, clé de la survie de l'ensemble du programme. La seconde faiblesse du système est l'éclatement des éleveurs en trois entités distinctes, sans stratégie commune, dès que l'État a allégé son soutien.

Le dysfonctionnement principal vient donc de la méconnaissance des marchés à l'export. Après le premier échec d'exportation en congelé, concurrencé à Rungis par les produits asiatiques à bas prix, l'État, l'Ifremer et la Saor décident de mettre leurs moyens en commun pour créer en urgence une filière $d^{\prime}$ exportation d'un produit entier, en frais, par avion, à destination de la grande distribution. L'aide d'un bureau d'études spécialisé dans le domaine de la commercialisation des fruits de mer conduit à choisir une stratégie de commercialisation basée sur la fraîcheur du produit parce qu'elle justifie un prix double de celui du produit d'Asie. La chaîne logistique «du bassin amazonien à l'hypermarché parisien » pour une crevette fraîche, sur glace, livrée en $24 \mathrm{~h}$, est organisée en un temps record grâce à une forte mobilisation de tous les acteurs. Contre toute attente, cette campagne "Crevette bleue des Caraibes » (1988-1989) est un succès : 88 tonnes vendues en frais en un an à un prix double du même produit venu d'Asie, ce dernier étant congelé (Lacroix et al., 1993).

Plus confiantes dans le produit et rassurées par le comportement des consommateurs (réachat après le premier achat de "curiosité »), les centrales d'achat françaises demandent alors la fiabilité et la régularité de fourniture de plusieurs tonnes de crevettes de Guyane par semaine avant tout référencement définitif dans leur catalogue d'offres. Mais une telle performance est encore hors de portée d'une filière jeune, fragile et dispersée, avec des infrastructures limitées et peu fiables pour le traitement local et le transport (deux avions Air France par semaine en vol direct Cayenne - Paris). En effet, comment livrer une tonne de crevettes en frais à l'aéroport quand la piste de latérite est coupée régulièrement en saison des pluies? Comment garantir une chaîne de froid fiable quand il n'existe que deux fabriques industrielles de glace pour tout le territoire?

Par ailleurs se pose le problème de la concurrence des importations d'Asie : est-ce que le label « Caraibe » et la fraîcheur de la crevette de Guyane suffiraient à terme pour conserver un avantage compétitif ? Un schéma de type néo-calédonien, avec le développement d'un marché local de plus de 500 tonnes, n'est pas envisageable en raison de la faiblesse du pouvoir d'achat local et de l'absence de tourisme. Cette situation aurait alors justifié un soutien fort des pouvoirs publics, voire de l'Europe, pour essayer de mettre en place une filière durable. Il faut noter ici que les scientifiques de l'Ifremer - et j'étais concerné au premier chef en tant que responsable de la partie du grossissement-, bien que cette phase du développement ne soit pas de leur responsabilité, vont 


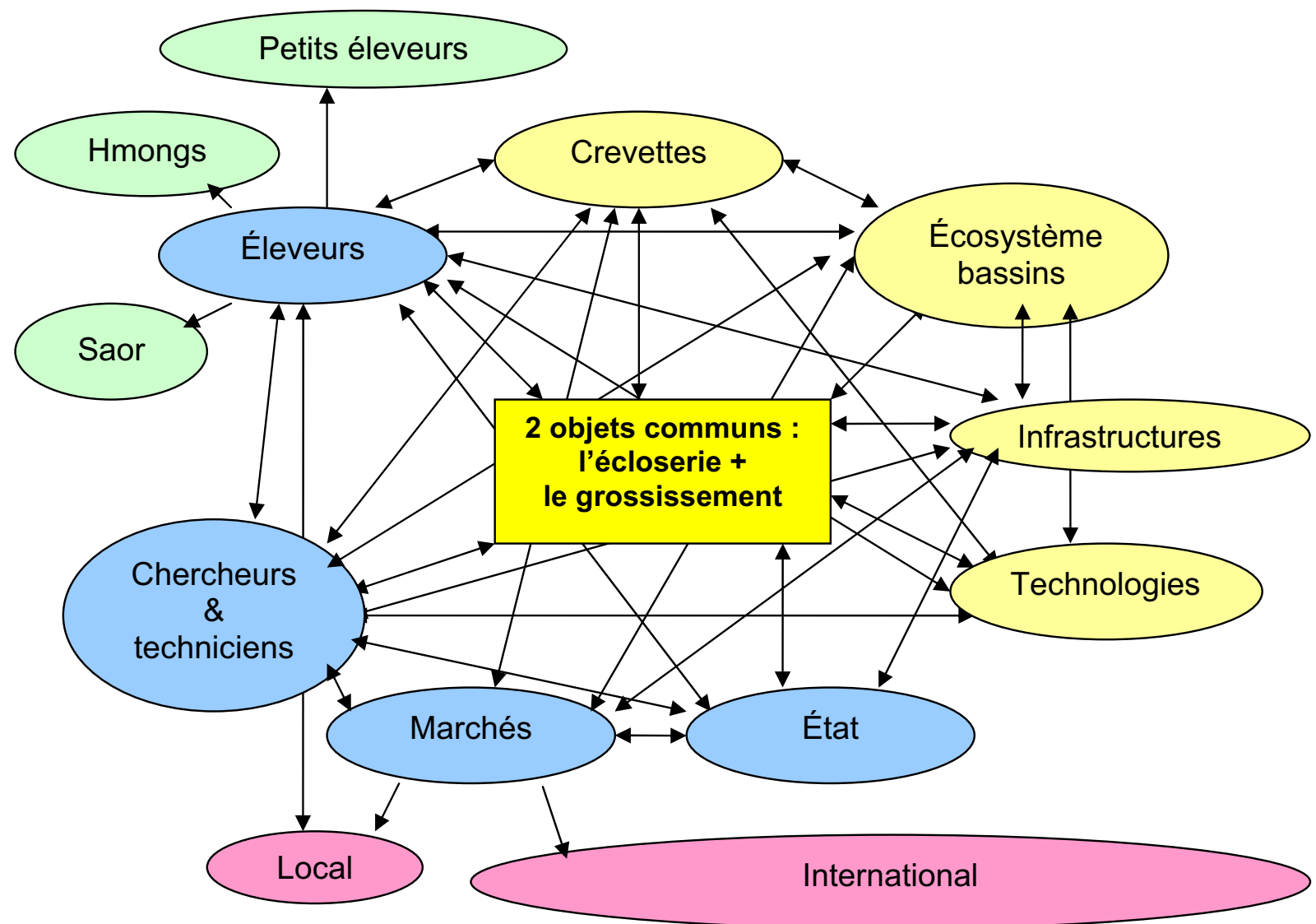

Fig. 2. Schéma du réseau d'acteurs appliqué au cas du développement de l'aquaculture de la crevette d'eau douce en Guyane française de 1982 à 1991.

s'engager sous diverses formes dans la mise en place de cette opération: mobilisation d'une expertise économique, participation à la mise en place d'un marketing adapté, appui aux pêches et à la logistique lors des jours de livraison à l'aéroport. À la station de l'Ifremer de Guyane, nous savions tous que si cette ultime voie de commercialisation échouait, nos efforts de recherche finalisée ne se justifiaient plus.

Dans ce contexte tendu de faible rentabilité des entreprises et de forte vulnérabilité aux aléas de transport, le coup de grâce est donné par la Saor qui «trahit » ses engagements de livraison de post-larves auprès de l'écloserie régionale. Alors que les commandes de la Saor en PL représentent $50 \%$ du chiffre d'affaires de l'écloserie, ses dirigeants décident en 1990 d'en construire une, persuadés qu'ils feraient des économies. Estimant que la filière se disperse au moment même où il est vital de se regrouper, l'État décide en 1990 d'arrêter tout soutien. J'ai alors reçu la mission d'organiser le démantèlement de l'opération, écloserie et station d'expérimentation de Kourou incluses, après dix années d'efforts, en prenant soin en priorité de trouver un emploi stable à tout le personnel local.
On peut légitimement poser la question du manque de réactivité du système par rapport aux problèmes rencontrés, surtout quand ceux-ci engagent la survie même de ce système. La saturation progressive des marchés locaux était pourtant aisément prévisible en comptabilisant les surfaces de bassins mises en eau ${ }^{14}$ et la programmation même du développement aisément orientable par les subventions à la mise en eau de bassins. Par ailleurs, la dynamique propre à un système complexe d'acteurs liés par un projet lancé par un leader bien identifié ${ }^{15}$ peut-elle survivre à un changement de portage au moment même où s'accumulent les difficultés ? Dans les deux cas, aux Antilles comme en Guyane française, se retrouve cette situation critique. Le lien entre les deux phénomènes apparaît clairement. Les transferts de responsabilités affaiblissent le réseau de relations des acteurs et donc réduisent leur réactivité par rapport aux problèmes qui se posent lors du changement de phase.

\footnotetext{
${ }^{14}$ Une capacité d'anticipation permettrait même de passer de la « réactivité » à la « proactivité ».

15 Et considéré comme responsable moral du projet lancé : les élus aux Antilles, l'État en Guyane.
} 
Les problèmes, surmontables dans la configuration initiale, deviennent insolubles dans une configuration nouvelle car solidarité et moyens s'affaiblissent au moment où il faudrait les renforcer. Le système devient alors encore plus vulnérable à toute « trahison » dont la légitimité et le risque d'occurrence s'accroissent avec l'effacement du leader initial ou le transfert de sa responsabilité.

On peut avancer que, dans le cadre inspiré par la théorie de l'acteur-réseau, déjà connue à cette période, une analyse des processus sociaux et de leur interaction avec les objets techniques aurait pu identifier les points de vulnérabilité du dispositif et notamment le risque majeur que constituait la construction d'une autre écloserie. Celle-ci devenait «mécaniquement » concurrente de l'écloserie régionale. Par ailleurs, tous les décideurs connaissaient la contradiction entre l'étroitesse du marché local et la capacité de production planifiée. Mais cette vision sous-entend deux conditions : un intérêt politique local, qui ne s'est jamais exprimé, à la différence des Antilles, et une volonté institutionnelle de développement sur le long terme, observée ni aux Antilles ni en Guyane.

\section{Conclusion pour la Guyane}

La question centrale, que je me pose depuis vingt ans, demeure : pourquoi est-on resté aveugle aussi longtemps en Guyane sur le problème du débouché commercial à terme ? La première explication est liée à la volonté de l'État de faire du développement en parallèle de la croissance du domaine spatial à Kourou. La planification du développement, notamment en matière d'aquaculture, est déjà considérée comme du ressort de l'État en métropole (Cacqueray, 2011); il l'est a fortiori en Guyane, où l'État joue un rôle premier depuis 1665 (Vignon, 1985 ; Anon, 2008). Quand les financements disponibles sont importants et que les propositions de projets sont peu nombreuses, la sélectivité qui en résulte est faible. Or, le programme de développement du Macrobrachium en Guyane s'appuie sur des prémisses apparemment solides : une technologie d'écloserie ayant fait ses preuves, des méthodes de grossissement robustes et simples d'application, une abondance d'eau douce et d'espaces plats facilement aménageables en bassins.

La seconde explication paraît de l'ordre du bon sens : il y a tellement d'incertitudes dans la faisabilité de cet élevage, notamment sur le plan de la biologie de cette espèce dans un environnement aussi particulier que celui de la Guyane, qu'il semble prématuré d'anticiper des problèmes de commercialisation. Satisfaire un marché local serait déjà considéré comme une réussite! Si cela fonctionne bien, il sera toujours temps de mettre en place une filière d'exportation adaptée.

La troisième raison est de l'ordre de l'implicite : la pêche industrielle de crevettes marines occupe une place économique importante en Guyane (25 millions/an dans les années 1990). C'est le troisième secteur économique de Guyane après le spatial et l'orpaillage, avec des centaines d'emplois en mer comme à terre. D'où l'idée, logique, que cette industrie solide saurait commercialiser aussi des crevettes d'eau douce. Mais les marchés correspondants n'ont rien à voir ; pire, l'image de la crevette d'eau douce est à créer ex nihilo avec trois handicaps: le goût (non salé), des pinces longues (encombrantes) et un céphalothorax massif mais non comestible (Griessinger et al., 1991).

Finalement, on pourrait dire que la filière Macrobrachium en Guyane n'a jamais "décollé " parce que les décideurs de son lancement n'ont jamais cru à l'éventualité de son succès. Aussi, ils n'ont pas estimé utile de mettre en place ce qui était indispensable pour passer d'une échelle artisanale pour un micromarché local à une échelle d'exportation pour des marchés internationaux normés. Pour assurer un flux d'exportation de plusieurs tonnes par semaine de produit frais à destination de Paris ou de Miami ${ }^{16}$, il fallait être capable de soutenir la création et la coordination de trois ou quatre fermes de la taille de la Saor afin de sécuriser la fourniture de crevettes, voire de faire face à des commandes spéciales au moment des pics d'achat comme à Noël. Il aurait fallu un opérateur unique pour la commercialisation d'un produit au marketing très ciblé. À l'évidence, aucun des acteurs n'avait le profil ni les compétences. Mais si cet opérateur unique avait été mis en place, est-ce que des « aventuriers de l'aquaculture » qui créent des fermes de crevettes au bout d'une piste de latérite en pleine forêt d'Amazonie auraient accepté de devenir de simples fournisseurs de produits calibrés selon des normes décidées à Rungis ? Si cette sorte d'entrepreneurs se rencontre encore en Guyane, ce n'est probablement pas pour alimenter la mondialisation du commerce des produits aquatiques.

Pour la part incombant à l'Ifremer, nous avions tenu nos engagements en matière d'écloserie comme de grossissement, avec des travaux de recherche finalisée, un effort de formation continue en Guyane et une valorisation de nos résultats au Brésil. En tant que chef de laboratoire, lors de la dernière année du projet, je coordonnais la synthèse de cette expérience de dix ans (Griessinger et al., 1991). Le livre qui en résulta était assez complet pour que la FAO le cite toujours comme référence dans la fiche Macrobrachium, mais la partie socioéconomique me laissa insatisfait parce qu'elle limitait

\footnotetext{
${ }^{16}$ Par le vol hebdomadaire Cayenne - Fort-de-France Pointe-à-Pitre - Porto Rico - Miami ; cette idée était assez réaliste pour qu'un commercial de la chaîne américaine de restaurants Red Lobster Inn, T. Royal, vienne en Guyane en 1988 évaluer la fiabilité d'un approvisionnement en "sweet giant prawn ».
} 
l'analyse aux critères de la rentabilité financière. Or je savais, d'expérience, que le contexte politique, les jeux $\mathrm{d}^{\prime}$ acteurs et la gouvernance avaient un poids au moins de même magnitude que le rendement des bassins et le prix sur le marché international. On aurait dû être capable de s'interroger sur la durabilité de cette filière : exporter par avion des crevettes entières à 7000 km du lieu de production pour un grand marché, certes, mais concurrentiel, n'était-ce pas irréaliste sur le long terme : trop de maillons, trop d'acteurs aux objectifs différents, trop peu de fiabilité dans la chaîne logistique et pas assez de rentabilité pour compenser les risques ? Cette question n'était pas formulable ainsi en 1990. Mais l'analyse prospective l'aurait peut-être fait émerger.

\section{Conclusion}

\section{Les limites de l'approche économique}

Il est utile de se demander d'abord si une analyse économique couplée à une réflexion de type "forces, faiblesses, opportunités et risques " n'aurait pas suffi à expliquer les deux situations. Concernant les Antilles, les situations similaires rencontrées à Hawaï, Maurice ou Porto Rico montraient des rentabilités économiques solides, dès lors que le maillon faible, l'écloserie, est maîtrisé et que le marché reste un marché de niche. Or, la fiabilité de l'écloserie est précisément le point fort de la méthode française et le marché antillais offre un positionnement naturel haut de gamme pour le Macrobrachium, produit déjà connu et apprécié sur place. En Guyane, l'analyse économique du projet de développement initial emprunte le modèle « crevette de mer » pour la commercialisation et s'appuie sur des économies d'échelle importantes en écloserie comme en grossissement. Mais si le prix de la poste-larve est assez prévisible (et il sera tenu), d'importantes incertitudes demeurent sur les rendements dans cet environnement amazonien complètement nouveau. Par ailleurs, les subventions en provenance de la France (contrats de plan État-Région) comme de 1'Union européenne (Fonds pour le développement régional, pour la pêche et l'aquaculture ; Fonds social européen) biaisent l'analyse économique en mettant l'investissement et l'emploi au premier plan des priorités. En effet, à qui attribuer l'entretien des pistes qui relient les fermes aquacoles à la route nationale? Doivent-elles être du ressort de l'État au titre de l'aménagement de «pénétrantes » facilitant l'accès à la forêt, ou doivent-elles être prises en charge par les aquaculteurs? La viabilité de la ferme aquacole de 10 ha, confiée à la coopérative des réfugiés Hmongs arrivés quinze ans plus tôt, ne peut-elle être analysée qu'en termes économiques ? Cette approche serait assez éloignée de la perception du développement des Hmongs (Géraud, 1997).
Cette difficulté d'utilisation de critères économiques dans des projets de développement innovants, comme en aquaculture, a été identifiée depuis longtemps (Pollnac et al., 1982). Ces critères donnent des éléments comparatifs intéressants, notamment quand les contextes climatiques ou d'infrastructures sont similaires, comme dans l'arc caraibe. Cependant, l'analyse multicritère menée dans quatre sites contrastés (Porto Rico, État de Rio, Antilles, Guyane) par quatre experts différents montre que les critères économiques n'apparaissent pas comme déterminants premiers dans le succès ou l'échec des projets aquacoles sur le court comme sur le moyen terme (Lacroix et al., 1993). De fait, au cours des trois phases de développement d'un projet aquacole de Macrobrachium, décrites dans le tableau 1 (test, développement, croisière), les facteurs déterminants du succès ou de l'échec concernent surtout «l'environnement» de la viabilité économique : qualité des infrastructures ; détermination des investisseurs; soutien institutionnel, financier et politique ; capacités d'adapter la technologie aux contraintes locales; acceptabilité sociale de ce nouvel élevage (consommateurs et producteurs).

Durant la phase de "croisière », les éléments déterminants sont la recherche et les hommes dans le sens d'un effort permanent de progrès de la technologie et de la valorisation commerciale. En conséquence, les auteurs concluaient en 1993 que la recherche finalisée en aquaculture a deux formes successives de priorités : d'abord, en collaboration avec les producteurs, elle doit faire progresser la technologie pour assurer la fiabilité de la production et sa rentabilité. Ensuite, dans une perspective de long terme, la recherche doit prendre en compte l'évolution des connaissances et des techniques et préparer les mutations dans un contexte international de plus en plus compétitif: nouvelles espèces, nouvelles techniques, nouveaux marchés. Cette analyse, classique en termes de méthode, est à relativiser en raison du profil des quatre experts notateurs, tous chercheurs ou ingénieurs. Or la propension des scientifiques à sous-estimer le poids des données économiques est connue (Cazes, 1986). Il aurait été intéressant de faire faire cette évaluation des facteurs-clés par plusieurs autres experts issus d'autres disciplines.

\section{L'apport de la sociologie de la traduction}

Si l'approche économique semble insuffisante pour éclairer la structuration et l'évolution de ces projets de développement, est-ce que l'approche de la sociologie de la traduction permet de construire un cadre d'analyse plus pertinent? Si l'analyse du développement est dans la compréhension des mécanismes réels mis en œuvre et des interactions sociales mises en mouvement (Olivier de Sardan, 2001), alors elle est légitime dans ces deux cas d'étude. Il s'agit ici d'aller plus loin qu'un simple constat 
Tableau 1. Étapes successives de la recherche et du développement pour l'élevage de la crevette géante d'eau douce Macrobrachium rosenbergii aux Antilles et en Guyane françaises de 1977 à 1990.

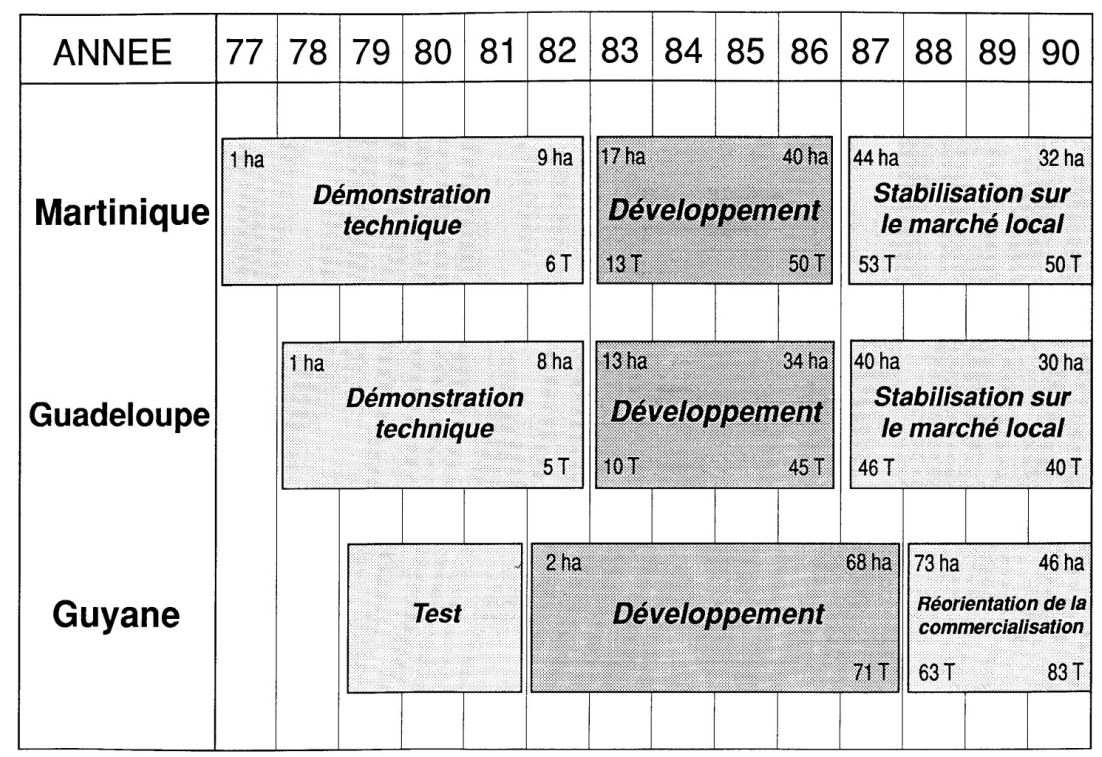

explicatif. L'intérêt de l'exercice est de comprendre comment une volonté initiale légitime de développement, qu'elle vienne de l'État, de collectivités locales ou d'un entrepreneur privé, peut aboutir à un échec et d'en tirer des reformulations utiles en termes de questionnement sociologique pour l'avenir. En effet, la même volonté de développement est toujours à l'œuvre dans l'Outre-mer français, notamment en aquaculture, et pour les mêmes raisons politiques qu'il y a trente ans, comme en Guyane (Anon, 2008) ou à Mayotte (projet de développement aquacole en cours). En d'autres termes, les causes des échecs de ces projets apparemment fondés, innovants et bien soutenus, auraient-elles pu être prévues ou identifiées plus tôt, dès la phase de planification par exemple?

L'approche de la sociologie de la traduction montre que le concept initial est toujours convaincant : l'intuition de J. Bally sur le développement du Macrobrachium aux Antilles sur le modèle de la réussite mauricienne, ou l'idée d'un élevage d'envergure de cette même espèce à l'échelle guyanaise après le succès initial aux Antilles. Le concept sur lequel se bâtit la légitimité du projet est donc solide et reconnu par tous les acteurs initiaux. Il ne manque pas alors de documents démontrant le bienfondé du projet et détaillant les étapes à respecter, les connaissances scientifiques et technologiques à acquérir ou compléter, les financements et les partenariats à mobiliser. La prise de risques apparaît alors à deux niveaux. Le premier concerne la prévision de l'évolution du marché, qui conditionne beaucoup de facteurs sur le moyen terme, notamment les capacités de transformation et de commercialisation. Or, le caractère fortement innovant du projet fait que les décideurs restent sur une image de marché de niche «durable» en reportant à plus tard l'organisation de la filière pour une ouverture à l'international, considérée comme hypothétique et lointaine dans le temps. Le second type de risque concerne l'organisation même de la filière car, dans les deux cas, c'est la dislocation des liens entre les acteurs qui entraîne le déclin de celle-ci.

Il n'y a pas de fatalité d'échec qui serait liée à l'introduction de l'aquaculture dans un milieu vierge, comme le montrent les succès des filières de pisciculture en Afrique de l'Ouest (Halftermeyer, 2009) ou de crevetticulture au Brésil (Rocha, 2004). Le point critique est celui du passage à la phase de croisière. C'est à ce moment, soit entre cinq et dix ans selon l'envergure du projet, les moyens consentis et le contexte géopolitique, que les points de faiblesse deviennent déterminants pour la survie à terme du projet (Denis et al., 2001). Les problèmes communs sont pourtant bien cernés : marché, évolution des technologies, organisation des acteurs et gouvernance. Mais ils se posent dans l'urgence parce qu'il n'y a pas eu d'anticipation du succès du projet. En Guyane, les autorités responsables du Plan vert n'ont pas prévu de répondre à une question simple : si « cela marche », comment placer 100 à 200 tonnes de crevettes par an sur un marché mondial d'un million de tonnes avec un coût de production deux fois supérieur au prix du marché ? Aux Antilles, les conseillers régionaux n'ont pas imaginé les conséquences d'un succès technique et commercial et n'ont donc pas prévu de structurer la production locale pour éviter les risques de dispersion des producteurs. Si ces deux éléments (marché, organisation) avaient été bien évalués dès le départ, aurait-on évité l'échec? Répondre affirmativement à une telle question reviendrait à recommander d'introduire d'autres éléments 
dans les dossiers d'instruction de projets comme une analyse fine des marchés potentiels, y compris à l'international, et un cadre plus rigide d'engagement des éleveurs, bloquant notamment la construction d'écloserie concurrente du projet collectif. Mais cette approche va à l'encontre des acquis de la sociologie de la traduction. Celle-ci démontre dans de nombreux cas que les acteurs ne sont pas des «pions » passifs qui appliqueraient un plan de développement prédéfini. Ils sont au contraire des éléments actifs d'un processus de traductions multiples d'intérêts, d'intéressement et d'influences (Jamieson, 2005 ; Lavigne Delville, 2011). En effet, aucun projet de développement, a fortiori pour un élevage d'une espèce animale récemment maîtrisée et dans un environnement naturel nouveau, ne peut prétendre lier ses chances de réussite à la rigueur et la précision d'une programmation prétendument exhaustive (Geis, 2010). Tout projet de développement inclut des incertitudes et celles-ci doivent être levées par les acteurs au fil des étapes du projet (Latour, 1992). En effet, les combinatoires des projets sont chaque fois en grande partie nouvelles avec des logiques techniques, économiques et politiques différentes (Olivier de Sardan, 1995). Cette observation a justifié chez divers auteurs l'éloge du « bricolage » (Lévi-Strauss, 1962) qui permet «un agencement original des éléments tirés de structurations englobantes et des considérations d'opportunité stratégiques » (Friedberg, 1993). On constate que l'État français privilégie encore aujourd'hui le cadre normatif comme le montre le plan actuel de développement de la Guyane (Anon, 2008), alors que les données sociologiques risquent d'être bouleversées si les forages exploratoires au large de Cayenne confirment la présence de pétrole exploitable.

\section{Que dire aujourd'hui avec le recul ?}

Les deux approches analytiques sont riches d'enseignements dans la relecture de ces deux projets de développement passés. Elles apparaissent complémentaires : la première décrit plutôt le cadre général du projet et la position initiale des acteurs; la seconde précise le système dynamique de tous les actants et permet d'anticiper les points de faiblesse, de déséquilibre ou de fracture du système. Elles constituent donc deux approches pour évaluer la robustesse du projet et la pertinence des choix stratégiques sur les sources de financement pérennes, le portage de l'initiative sur le long terme, le ou les marchés visés, les besoins en recherche finalisée et en formation, etc. Dans les deux cas, il s'agit d'un processus dynamique multiacteur, mais positionné dans un plan normatif décidé pour dix ans, avant que les problèmes de marché ne deviennent aigus. Les mouvements de tous les acteurs ont leur logique interne, mais l'effritement des liens et la disparition des objets collectifs condamnent les projets sur le moyen terme. Enfin, la dilution finale des responsabilités ne permet pas l'apprentissage des leçons de l'échec.

Au-delà des diagnostics des échecs, il persiste une interrogation sur le moteur même des projets : l'initiative politique. Dans les deux cas, j'ai été amené à participer à l'élaboration des projets et à leur orientation, en liaison avec les élus et l'administration. Chaque fois, une forte conviction politique est à l'origine de la mobilisation de nombreux acteurs, de financements importants et sur une dizaine d'années. C'est aussi l'arrêt du soutien des politiques qui marque le début du déclin des programmes en cours. Les questions pertinentes relèveraient-elles finalement des sciences politiques ? De fait, si l'on se borne à une analyse de type économique, technologique ou sociologique des deux projets, peut-on dépasser «la chronique d'une mort annoncée »? Les clés d'une meilleure chance de succès ne seraient-elles pas dans la capacité de tirer des leçons des échecs précédents, de prendre le temps de formuler collectivement un projet de long terme, de former les décideurs aux postes importants, de mettre en place un dispositif d'indicateurs permettant d'éviter l'écueil récurrent de l'absence de réactivité ?

Dans toutes ces propositions revient un facteur commun, le temps. Est-ce qu'en dernier ressort, ce ne serait pas sa maîtrise qui ferait défaut? Mais comment intégrer ce facteur car les acteurs n'ont pas la même échelle de temps. Leur incompatibilité relative est source de problèmes récurrents et d'incompréhension mutuelle. De fait, on oppose classiquement les arbitrages fondés sur le court terme, qui sont supposés viser le concret, le pragmatique, le "sûr ", et les arbitrages fondés sur le long terme, qui sont censés sélectionner les solutions plus durables et plus "stratégiques », mais dont la probabilité d'occurrence est plus aléatoire. Une des justifications de la prospective est précisément de réduire le champ des incertitudes sur le long terme. Il existe de nombreuses méthodes de simulation du temps pour chaque discipline mobilisée dans ce type de projet : la compensation de l'usure, de l'obsolescence et le progrès des connaissances dans les domaines techniques et scientifiques. La modélisation financière et économique met à disposition des décideurs et des bailleurs de fonds une batterie d'indicateurs connus, comparables à ceux d'autres projets plus anciens. La sociologie dispose aussi de divers moyens pour décrire les dynamiques en action et donner des indications sur les évolutions les plus probables sur le moyen terme. La prospective peut mobiliser des méthodes variées, éprouvées depuis une vingtaine d'années, notamment la méthode des scénarios (Godet, 1991 ; Hatem et al., 1993,). Mais toutes ces démarches sont le plus souvent parallèles parce qu'elles relèvent d'expertises disciplinaires. Les experts ne croisent donc pas leurs savoirs. En général, c'est le pouvoir politique qui détient, avec l'administration, les divers éléments d'analyse et de 
discussion (Theys, 2003). C'est précisément l'intégration de ces éléments qui permettrait, dans un premier temps, de distinguer les scénarios possibles, et, dans un second temps, d'identifier les conséquences de chaque scénario sur les composantes de la durabilité. Cette approche éviterait le risque de dichotomie entre une prospective de type «modélisation des indicateurs quantitatifs » et une prospective de type «scénarios » (Cazes, 1986 ; Van der Heijden, 1996). Y. Stourdzé (1987) développe la métaphore entre l'accélération de l'évolution des sociétés et le pilotage d'avions de plus en plus rapides. Il observe qu'il faut deux éléments nouveaux pour réduire les risques d'écrasement pour un avion : des indicateurs pertinents et un simulateur permettant de « rejouer » de multiples situations de vol afin d'acquérir la sécurité en vol réel. Dans le cas d'un projet de développement risqué, comme l'aquaculture d'outre-mer par exemple, l'intégration des indicateurs, issus des expertises disciplinaires, dans des simulations de futurs potentiels permettrait alors de mieux discerner les forces et faiblesses d'un projet de développement, ou ses variantes, à des échelles de temps variables. L'échelon politique garderait sa prééminence, mais les fondements de ses choix pourraient être explicités en réduisant les marges d'incertitude. Une telle approche pourrait entrer en dialogue avec la sociologie du développement, notamment parce que cette discipline dispose déjà d'outils pour décrypter des situations à forte variabilité. Elle faciliterait ainsi le dialogue entre disciplines et «l'hybridation» des savoirs au service d'un objectif commun et appliqué, objectif dans lequel chaque acteur aurait une meilleure visibilité (Passet et Vivien, 2011). Une telle démarche ne se limiterait pas à une meilleure appropriation du projet de développement; elle améliorerait aussi la responsabilisation des parties prenantes dès le stade de la conception du projet et faciliterait la maîtrise de son évolution au fil des années. Cette vision, en phase avec les principes de la durabilité (Brundtland, 1987), ouvre un nouveau champ d'analyse, dans le cadre ex ante (prospective) autant que dans le cadre ex post. Mais si les aspects techniques de la scénarisation ont été déjà explorés, il reste à enrichir l'analyse au plan sociologique, avec un équilibre à trouver entre l'inductif et le déductif.

\section{Références}

Alcamo, J. (Ed.), 2008. Environmental futures: The practice of environmental scenarios analysis, Amsterdam, Elsevier.

Alter, N., 2000. L'Innovation ordinaire, Coll. Quadrige, Paris, Puf.

Anon, 2008. La Guyane, terre fertile : programme de développement rural de la Guyane 2007-2013. Diagnostic et stratégie, mesures, données financières, gestion de programme. Préfecture, conseil régional, conseil général, CNES, ONF, Cirad, UE.
Aquacop, 1977. Macrobrachium rosenbergii (de Man) culture in Polynesia: Progress in developing a mass intensive larval rearing technique in clear water. $8^{\text {th }}$ Workshop of World Mariculture Society, Costa Rica, Jan. 10-13. Published in Aquaculture en milieu tropical, Brest, Ifremer, 1984, 46-62.

Barré, R., 2000. Le foresight britannique, un nouvel instrument de gouvernance?, Futuribles, 249, 5-24.

Blanc-Pamard, C., Fauroux, E., 2004. L'illusion participative : exemples ouest-malgaches, Autrepart, 3, 31, 3-19, doi: 10.3917 /autr.031.0003.

Brundtland, H.G., 1987. Notre futur commun, Oxford, Oxford University Press.

Callon, M., 1986. Éléments pour une sociologie de la traduction : la domestication des coquilles Saint-jacques et des marins pêcheurs en baie de Saint-Brieuc, L'Année Sociologique, 36, 169 -207.

Callon, M., Lascoumes, P., Barthe, Y., 2001. Agir dans un monde incertain: essai sur la démocratie technique, Coll. La couleur des idées, Paris, Le Seuil.

Cacqueray, M. (de), 2011. La planification des espaces maritimes en France métropolitaine : un enjeu majeur pour la mise en cuvre de la gestion intégrée de la mer et du littoral. Thèse de doctorat de géographie, Laboratoire Géomer, UBO, Université européenne de Bretagne.

Cazes, B., [1986] 2008. Histoire des futurs : les figures de l'avenir de saint Augustin au XXI siècle, Coll. Prospective, Essais et recherches, Paris, L'Harmattan.

Chevassus-au Louis, B., 2007. L'Analyse des risques : l'expert, le décideur et le citoyen, Coll. Sciences en question, Versailles, Éditions Quae.

Claes-Mekdade, C., 2006. La participation environnementale à la française : le citoyen, l'État et le sociologue, VertigO, la revue électronique en sciences de l'environnement, 7, 3 (http: / / vertigo.revues.org/8446).

Cornish, E., 2004. Futuring: The Exploration of the Future, Bethesda (MD), World Future Society.

Da Silva, N.J.R., Beuret, J.E., Mikolasek, O., Fontenelle, G., Dabbadie, L., Espagnoli, M.I., 2005. Dynamiques de développement de la pisciculture et politiques publiques dans la vallée du Ribeira, État de Sao Paulo (Brésil), Cahiers Agricultures, 14, 1, 59-63.

Denis, J., Hénocque, Y., Antona, M., 2001. Des outils et des hommes pour une gestion intégrée des zones côtières: guide méthodologique, Vol. 2, Paris, Unesco, COI.

Dherse, J.-L., Minguet, D.H., 1998. L'Éthique ou le Chaos, Paris, Presse de la Renaissance.

FAO, 2008. Sustainable growth and expansion of aquaculture: An ecosystem approach, FAO State of the World Fisheries and Aquaculture, 76.

FAO, 2010. Situation mondiale des pêches et de l'aquaculture, Rome, FAO (http://www.fao.org/docrep/013/i1820f/ i1820f.pdf).

Friedberg, E., 1993. Le Pouvoir et la Règle : dynamiques de l'action organisée, Coll. Sociologie, Paris, Le Seuil.

Geis, T., 2010. Transforming realities: Integrated coastal zone management, sustainability and connective aesthetics. Thesis of Master of resource management degree in coastal and marine management, University of Akureyri, Iceland. (http://skemman.is/stream/get/1946/5673/16957/1/ Tanja_Geis_Master_Heild.pdf). 
Géraud, M.O., 1997. Regards sur les Hmongs de Guyane française : les détours d'une tradition, Paris, L'Harmattan.

GFCM, 2010. Regional synthesis of the Mediterranean marine finfish aquaculture sector and development of a strategy for marketing and promotion of Mediterranean aquaculture, GFCM Studies and Reviews, 88, FAO, Rome.

Godet, M., 1991. L'Avenir autrement: manuel de prospective stratégique (tome 1 : Une indiscipline intellectuelle, tome 2: L'Art et la Méthode), Paris, Armand Colin.

Godet, M., 1997. De l'anticipation à l'action : manuel de prospective et de stratégie, Paris, Dunod.

Griessinger, J.M., Lacroix, D., Gondouin, P., 1991. L'élevage de la crevette tropicale d'eau douce, Plouzané, Ifremer.

Halftermeyer, S., 2009. Construire un réseau de producteurs ruraux autour d'une nouvelle production : l'exemple du projet piscicole de Guinée forestière (PPGF), Traverses, 32, APDRAF/Groupe Initiatives (http://www.apdra.org/ IMG/file/Articles/Traverse32.pdf).

Hatem, F., Cazes B., Roubelat, F., 1993. La Prospective : pratiques et méthodes, Coll. Gestion, Paris, Economica.

Hénocque, Y., Billé, R., 2005. Gestion intégrée du littoral. Communication au colloque international Prospective $d u$ littoral pour les générations futures, Ministère de l'Écologie et $\mathrm{du}$ Développement durable (MEDD), $1^{\mathrm{er}}-2$ mars, Paris (http:/ / www.iddri.org/Iddri/Equipe/gestionint-greRB.pdf).

Jamieson, D., 2005. Adaptation, mitigation and justice, in Perspectives on Climate change: Science, Economics, Politics, Ethics, Amsterdam, Oxford, Elsevier, 217-248.

Jouvenel, H. (de), 2004. Invitation à la prospective (An invitation to foresight), Coll. Perspectives, Paris, Futuribles.

Kumar, S., Corbridge, S., 2002. Programmed to fail?: Development projects and the politics of participation, Journal of Development Studies, 39, 2, 73-103.

Lacroix, D., Griessinger, J.M. Falguière, J.C., Pollet, T., 1987. Macrobrachium rosenbergii culture in the French West Indies and French Guiana: Validity of the continuous grow-out system as a mean of development. Communication à Caribbean Aquaculture Expo 87, Sept. 23-27, San Juan, Puerto Rico, in Chavez, C. (Ed.), El cultivo del camaron, langostin, cangrejo en el mundo: bases y technologias (http:// archimer.ifremer.fr/doc/00040/15170/12507.pdf).

Lacroix, D., Maubras, Y., 1991. L'expérience de l'exportation de la chevrette Macrobrachium rosenbergii en Guyane Française, Équinoxe, 35, 4-12.

Lacroix, D., Glude, J., Thomas, J.E., Le Menn, H., 1993. From research to commercialisation: Lessons from four different strategies in the development of freshwater prawn culture (Macrobrachium rosenbergii) in America since 1977, World Aquaculture Magazine, 25, 1, 4-17.

Latour, B., 1992. Aramis ou l'amour des techniques, Paris, La Découverte.

Lavigne Delville, P., 2011. Vers une socio-anthropologie des interventions de développement comme action publique. Mémoire d'HDR, Crea, Université Lyon II, Lyon.
Le Galès, P., 2006. Gouvernement et gouvernance des territoires, Problèmes Politiques et Sociaux, 922, La Documentation française.

Lévi-Strauss, C., 1962. La Pensée sauvage, Coll. Agora, Paris, Plon.

Marris, C., 2001. La perception des OGM par le public : remise en cause de quelques idées reçues, Économie Rurale, 266, 5879.

Mendras, H., Forsé, M., 1983. Le Changement social : tendances et paradigmes, Coll U, Paris, Armand Colin.

Mermet, L. (Ed.), 2005. Étudier les écologies futures : un chantier ouvert pour les recherches prospectives environnementales, Coll. Écopolis, Bruxelles, Éditions PIE - Peter Lang.

Noiville, C., Gouyon, P.-H., 2000. Principe de précaution et organismes génétiquement modifiés: le cas du maïs transgénique, in Kourilsky, P., Viney, G., Le Principe de précaution, Paris, Odile Jacob, 277-340.

Olivier de Sardan, J.-P., 1995. Anthropologie et développement: essai en socio-anthropologie du changement social, Marseille, Paris, Apad, Karthala.

Olivier de Sardan, J.-P., 2001. Les trois approches en anthropologie du développement, Revue Tiers Monde, 42, 168, 729764.

Passet, R., 1979. L'Économique et le Vivant, Paris, Payot.

Passet, R., Vivien, F.D., 2011. René Passet : la quête d'une bioéconomie transdisciplinaire, Natures Sciences Sociétés, 19, 4, 410-421.

Pollnac, R., Peterson, S., Smith, L., 1982. Elements in evaluating success and failure in aquaculture projects, in Smith, L.J., Peterson, S. (Eds.), Aquaculture Development in Less Developed Countries: Social Economic and Political Problems, Boulder (CO),Westview press, 131-143.

Rey-Valette, H., Roussel, S., 2006. L'évaluation des dimensions territoriale et institutionnelle du développement durable : le cas des politiques de gestion intégrée des zones côtières, Développement Durable et Territoires, Dossier 8 (http:// developpementdurable.revues.org/3311).

Rocha, I.P., 2004. Impactos socioeconômicos e ambientais da carcinicultura brasileira: mitos e verdades, Revista de ABCC, 7, 4, 29-36.

Rogers, E., 1983. Diffusion of Innovations, New York, Free Press. Schwartz, P., [1991] 2007. The Art of Long View: Planning for the Future in an Uncertain World, Chichester (UK), John Wiley \& sons Ltd.

Strourdzé, Y., 1987. Pour une poignée d'électrons, Paris, Fayard.

Theys, J., 2003. La gouvernance, entre innovation et impuissance, Développement Durable et Territoires, Dossier 2 (http:/ / developpementdurable.revues.org/1523).

UICN, 2007. Aquaculture and environment in Mediterranean aquaculture, in Guide for the sustainable development of Mediterranean aquaculture, Gland (CH), UICN.

Van der Heijden, K., 1996. Scenarios: The Art of Strategic Conversation, Chichester (UK), John Wiley and sons Ltd.

Vignon, R., 1985. Gran man baka (Journal d'un préfet de Guyane 1947-1976), Éditions Davol.

Reçu le 10 novembre 2011. Accepté le 3 mai 2013. 